\title{
Distributed Parametric Resonator: A Passive CMOS Frequency Divider
}

\author{
Wooram Lee, Student Member, IEEE, and Ehsan Afshari, Member, IEEE
}

\begin{abstract}
We present an electrical distributed parametric oscillator to realize a passive CMOS frequency divider with low phase noise. Instead of using active devices, which are the main sources of noise and power consumption, an oscillation at half of the input frequency is sustained by the parametric process based on nonlinear interaction with the input signal. To show the feasibility of the proposed approach, we have implemented a 20-GHz frequency divider in a $0.13-\mu \mathrm{m}$ CMOS process. Without any dc power consumption, 600-mV differential output amplitude is achieved for an input amplitude of $600 \mathrm{mV}$. The input frequency ranges from 18.5 to $23.5 \mathrm{GHz}$ with varactor tuning. The output phase noise is almost $6 \mathrm{~dB}$ lower than that of the input signal for all offset frequencies up to $1 \mathrm{MHz}$. There is a good agreement among analysis, simulation, and $10-\mathrm{MHz}$ measurement results. To the best of our knowledge, this is the first passive frequency divider in a CMOS process.
\end{abstract}

Index Terms-Dispersion, distributed system, frequency divider, low phase noise, low-power design, nonlinear capacitor, parametric amplification, phase matching.

\section{INTRODUCTION}

$\mathbf{T}$ HE frequency divider is an essential block in phase-locked loops (PLLs) and frequency synthesizers. The design of the frequency-divider block in any system is critical, since it consumes a large portion of the overall system power and it is one of the key contributors to the phase noise. The design of the frequency divider becomes even more challenging at high frequencies due to the limited speed of digital gates in a conventional digital frequency divider [1]. At these frequencies, an injection-locked frequency divider has been a good candidate due to its high speed and low power consumption [2]-[4]. Although there have been several works to further minimize the power consumption of the injection-locked frequency divider, the use of transistors for sustaining oscillation limits these efforts. Moreover, the channel noise caused by transistors degrades the output phase noise at a large offset frequency or near the edge of the locking range [5]-[8].

To overcome these limits, we use parametric amplification in which the nonlinear interaction with a pump results in the signal gain by transferring the energy from the pump to the signal [9], [10]. Since this method does not use any active devices, low

Manuscript received December 07, 2009; revised May 21, 2010; accepted May 29, 2010. Date of current version August 25, 2010. This paper was approved by Associate Editor Jacques Rudell. This work was supported in part by the C2S2 Focus Center, one of six research centers funded under the Focus Center Research Program (FCRP), a Semiconductor Research Corporation entity, and also by the National Science Foundation under CAREER Award 0954537. The work of W. Lee was supported by a Samsung fellowship.

The authors are with the School of Electrical and Computer Engineering, Cornell University, Ithaca, NY 14850 USA (e-mail: w1287@ cornell.edu).

Color versions of one or more of the figures in this paper are available online at http://ieeexplore.ieee.org.

Digital Object Identifier 10.1109/JSSC.2010.2056833 noise performance can be achieved [9]. However, parametric amplification has rarely been implemented as an amplifier in CMOS technology because it requires high quality factor inductors and varactors for sufficient gain. On the other hand, parametric oscillator, which is a harmonic oscillator sustained by the parametric amplification, can be CMOS compatible since it only needs enough gain to compensate the resonator loss. The oscillation frequency in the parametric oscillator is synchronized to one of the subharmonics of the pump signal. This phenomenon can be exploited to make a frequency divider. There have recently been a few works on parametric frequency dividers on printed circuit boards (PCBs). However, the operation frequency is limited to $2 \mathrm{GHz}$, and these designs are not CMOS-compatible.[11]-[13].

In this paper, we use the concept of parametric oscillation to design the first passive $20-\mathrm{GHz}$ CMOS frequency divider. It consists of two parallel nonlinear transmission lines using MOS varactors, forming a reflective distributed resonator. Its operation principle is the electronic analogue to that of the optical parametric oscillator [14], [15]. This distributed structure enables a stable startup condition and a broad range of frequency tunability. Moreover, the reflective resonator minimizes the number of components and forms a standing wave, which suppresses the pump signal at the output while maintaining a large output signal amplitude. In addition to zero static power consumption, the proposed frequency divider shows better phase-noise performance even at high offset frequencies since it does not have the channel noise from the transistors.

The remainder of this paper is organized as follows. Section II explains the basic concept of the distributed parametric amplification. Section III discusses the theoretical analysis of parametric oscillation, the standing wave formation of the reflective resonator, and a unique input-matching characteristic. Section IV discusses the phase-noise performance and compares it with the injection-locked frequency divider. Section V summarizes the design procedure and the measurement results. Finally, Section VI compares the proposed divider with other dividers with no static power consumption and its applications.

\section{Distributed PARAMETRIC AMPLifiCATION}

Assume a uniform artificial transmission line consisting of inductors and voltage-dependent (and hence nonlinear) capacitors shown in Fig. 1. By applying KCL at node $k$, whose voltage with respect to ground is $V_{k}$, and applying $\mathrm{KVL}$ across the two inductors connected to this node, one can easily show the voltages of adjacent nodes on this transmission line are related via

$$
l \frac{d}{d t}\left[c\left(V_{k}\right) \frac{d V_{k}}{d t}\right]=V_{k+1}-2 V_{k}+V_{k-1} .
$$




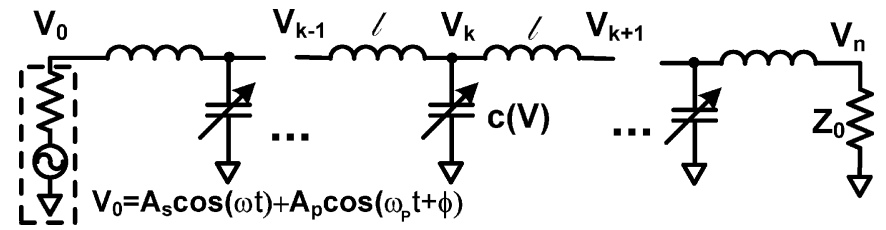

Fig. 1. Uniform nonlinear transmission line for parametric amplification.

The nonlinear capacitor is approximated with a first-order function

$$
c(V)=c_{0}(1+b V)
$$

where $c_{0}$ is the capacitance at zero bias and $b$ is the $\mathrm{C} / \mathrm{V}$ slope. For now, we neglect the loss of the transmission line. Equation (1) can be written as a partial differential equation

$$
\begin{aligned}
\frac{V_{k+1}-2 V_{k}+V_{k-1}}{h^{2}} & =\left[\frac{\left(V_{k+1}-V_{k}\right)}{h}-\frac{\left(V_{k}-V_{k-1}\right)}{h}\right] \frac{1}{h} \\
& \simeq \frac{\partial^{2} V}{\partial x^{2}} \\
& =L \frac{\partial}{\partial t}\left[C(V) \frac{\partial V}{\partial t}\right]
\end{aligned}
$$

by approximating the node voltage as a continuous variable over distance: $V_{k}=V(k h) \simeq V(x)$. Here, $h$ is the spacing between two adjacent nodes and $k$ is the section number. We also introduce a unit length inductance $L=l / h$ and a unit length capacitance $C=c / h$. For simplicity, we assume that the dispersion effect caused by discreetness is negligible, due to a small $h$ compared with the signal wavelength.

Now, pump and signal are applied to the left end of the transmission line, and the pump frequency $\left(\omega_{p}=2 \omega\right)$ is set to be twice the signal frequency $(\omega)$. By inserting $V=V_{s}+V_{p}$ and (2) into (3), (3) becomes

$$
\begin{aligned}
& \frac{\partial^{2}\left(V_{s}+V_{p}\right)}{\partial x^{2}}=L C_{0} \frac{\partial^{2}\left(V_{s}+V_{p}\right)}{\partial t^{2}} \\
& +L C_{0} b \frac{\partial^{2}\left(V_{s} \cdot V_{p}\right)}{\partial t^{2}}+\frac{L C_{0} b}{2}\left[\frac{\partial^{2}\left(V_{s} \cdot V_{s}\right)}{\partial t^{2}}+\frac{\partial^{2}\left(V_{p} \cdot V_{p}\right)}{\partial t^{2}}\right]
\end{aligned}
$$

where $V_{s}$ and $V_{p}$ are signal and pump voltages, respectively. The first term on the right corresponds to the linear wave propagation, and the second term represents the nonlinear coupling between pump and signal, which results in the parametric amplification. The third term on the right corresponds to the secondorder harmonic generation (SHG) for signal and pump. We assume the signal amplitude is so small that the SHG for the signal is negligible, and that the SHG for the pump is sufficiently suppressed since the cut-off frequency of the transmission line is set to be lower than the second harmonic of the pump frequency. With these assumptions, we simplify (4) as

$$
\frac{\partial^{2}\left(V_{s}+V_{p}\right)}{\partial x^{2}}=L C_{0} \frac{\partial^{2}\left(V_{s}+V_{p}\right)}{\partial t^{2}}+L C_{0} b \frac{\partial^{2}\left(V_{s} \cdot V_{p}\right)}{\partial t^{2}} .
$$

From coupled-mode theory [16], [17], the signal is defined as

$$
\begin{aligned}
& V_{s}(x, t)=V(x) e^{j \omega t}+V^{*}(x) e^{-j \omega t} \\
& V_{s}(0, t)=A_{s} \cos (\omega t)
\end{aligned}
$$

where $V(x)=A(x) e^{-j \beta x}, V^{*}(x)=A^{*}(x) e^{j \beta x}$, and $\beta$ is the signal propagation constant. Here, "**" denotes the complex conjugate. $A(x)$ and $A^{*}(x)$ are slowly varying functions over $x$. In other words, $\partial A / \partial x \ll \beta A$.

For a simple case, the pump is a sinusoidal function at a frequency of $\omega_{p}=2 \omega$. The initial phase difference between signal and pump is $\phi$ at $x=0$. Then, the pump can be written as

$$
\begin{aligned}
V_{p}(x, t) & =A_{p} \cos \left(\omega_{p} t-\beta_{p} x+\phi\right) \\
& =\frac{1}{2} A_{p}\left[e^{j\left(\omega_{p} t-\beta_{p} x+\phi\right)}+e^{-j\left(\omega_{p} t-\beta_{p} x+\phi\right)}\right]
\end{aligned}
$$

where $A_{p}$ is the amplitude of the pump and $\beta_{p}$ is the pump propagation constant. By substituting (6) and (9) into (5), and by approximating $\beta$ as $\beta \simeq \omega \sqrt{L C_{0}} \simeq \beta_{p} / 2$ (small dispersion assumption), we derive the active coupled-mode equations for $A(x)$ and $A^{*}(x)$ as

$$
\begin{aligned}
\frac{\partial A(x)}{\partial x} & =-\frac{j \beta b A_{p}}{4} A^{*}(x) e^{j \phi} \\
\frac{\partial A^{*}(x)}{\partial x} & =\frac{j \beta b A_{p}}{4} A(x) e^{-j \phi} .
\end{aligned}
$$

Combining (10) and (11), we will have

$$
\frac{\partial^{2} A(x)}{\partial x^{2}}=\left(\frac{\beta b A_{p}}{4}\right)^{2} A(x) .
$$

By applying the boundary condition of the signal in (7), and from (10) and (12), the complete solution of (12) is obtained as

$V_{s}(x, t)=A_{s}[\cosh s x \cdot \cos (\omega t-\beta x)+\sinh s x \cdot \sin (\omega t-\beta x+\phi)]$

where $s=\beta b A_{p} / 4$. Depending on the initial phase difference between pump and signal $\phi$, the signal gain can be exponentially growing or decaying over $x$ :

$$
G(x)=\frac{\left|V_{s}(x, t)\right|}{\left|V_{s}(0, t)\right|}=\left\{\begin{array}{ll}
\exp (s x), & \text { for } \phi=\frac{\pi}{2}(\max .) \\
\exp (-s x), & \text { for } \phi=-\frac{\pi}{2}(\min .)
\end{array} .\right.
$$

From (14), the maximum parametric gain for the $k$ th section is approximated by

$$
\begin{aligned}
G_{k} & =\left.G(k h)\right|_{\phi=\pi / 2} \\
& =\exp \left(\frac{b A_{p}}{4} \cdot \omega \sqrt{\left(\frac{l}{h}\right)\left(\frac{c_{0}}{h}\right)} \cdot k h\right) \\
& =\exp \left(\frac{b A_{p} \omega \sqrt{l c_{0}}}{4} \cdot k\right) .
\end{aligned}
$$

Fig. 2 shows the calculated gain based on (15). The gain increases exponentially with the nonlinear factor, $b A_{p}$, as well as the section number. The nonlinear factor is defined by the multiplication of the varactor C/V slope $b$, and the pump amplitude $A_{p}$. Note that $b$ is 0.5 to $1.2 \mathrm{~V}^{-1}$ for an accumulation-mode nMOS varactor in a typical $0.13-\mu \mathrm{m}$ CMOS process [18]. It is interesting that the gain grows exponentially in space while in the conventional distributed amplifier it increases linearly. This type of amplification process where the pump frequency is exactly twice the signal frequency is called degenerate parametric amplification.

In addition, (15) shows that the gain increases with the inductance and the average capacitance at a given signal frequency. 


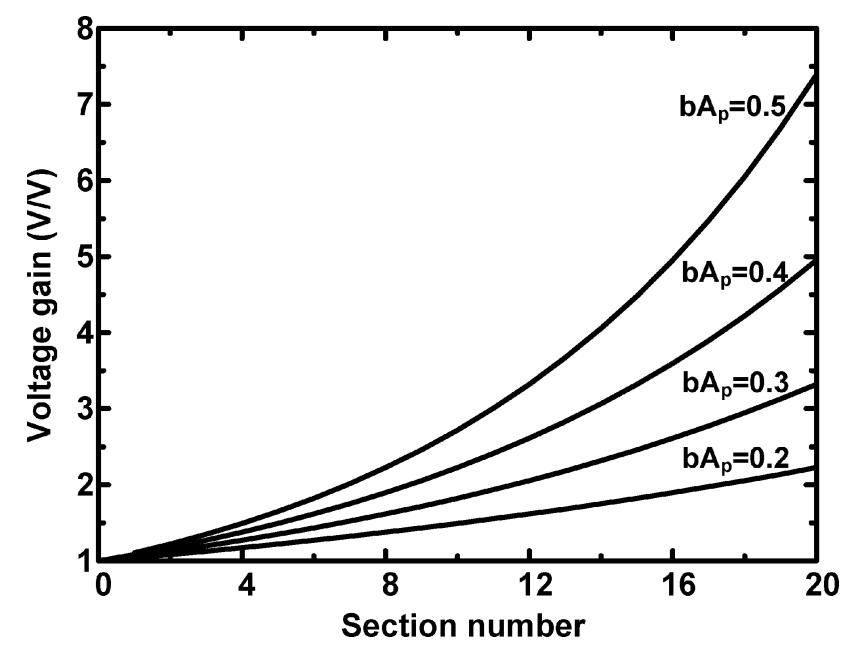

Fig. 2. Maximum degenerate parametric gain versus section number with different nonlinearity factors $b A_{p}$ for $\omega=10 \mathrm{GHz}\left(l=630 \mathrm{pH}, c_{0}=250 \mathrm{fF}\right)$.

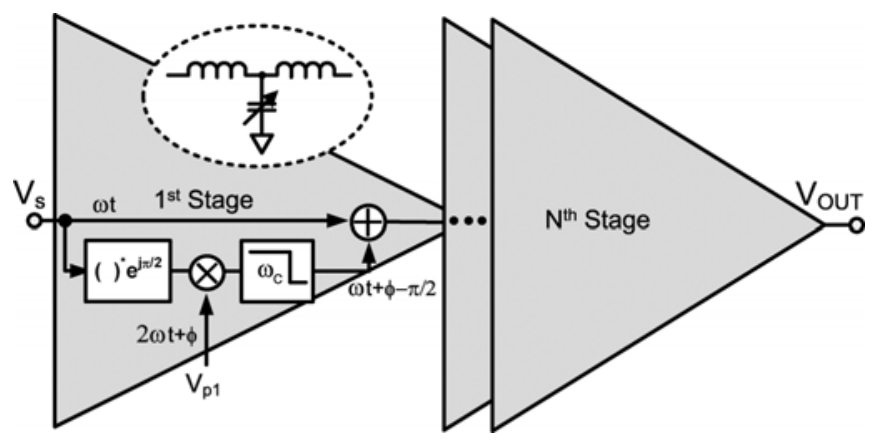

Fig. 3. Illustration of distributed parametric amplification using a series of cascaded amplifiers.

Intuitively, this is due to the fact that, in a transmission line with larger inductance and capacitance, the pump and the signal stay longer within each section, increasing the energy transfer. In other words, the effective propagation length is increased. However, the increase in the inductance and capacitance also leads to a decrease in the cutoff frequency defined by $\omega_{c}=$ $2 / \sqrt{l c_{0}}$. The pump amplitude decreases as the cutoff frequency approaches the pump frequency. Therefore, the cutoff frequency of the transmission line should be optimized for maximum gain.

Fig. 3 illustrates the principle of distributed parametric amplification using a series of cascaded amplifiers. The nonlinear interaction of the signal $(\omega t)$ and the pump $(2 \omega t+\phi)$ is shown using a mixer. This interaction generates two components at $(\omega t+\phi-\pi / 2)$ and $(3 \omega t+\phi+\pi / 2)$. However, $(3 \omega t+\phi+\pi / 2)$ is suppressed due to the cutoff frequency of the transmission line. Therefore, only $(\omega t+\phi-\pi / 2)$ remains, and it is added to the signal constructively or destructively depending on $\phi$. The resulting signal enters the next section, with an exponential amplification or attenuation (depending on the initial phase difference, $\phi)$. The main advantage of parametric amplification is that it does not require any active devices, which gives it the potential for much lower noise figure than conventional transistor-based amplifiers.

So far, we assumed that there is no dispersion in the transmission line for simple analysis. The pump propagation constant,
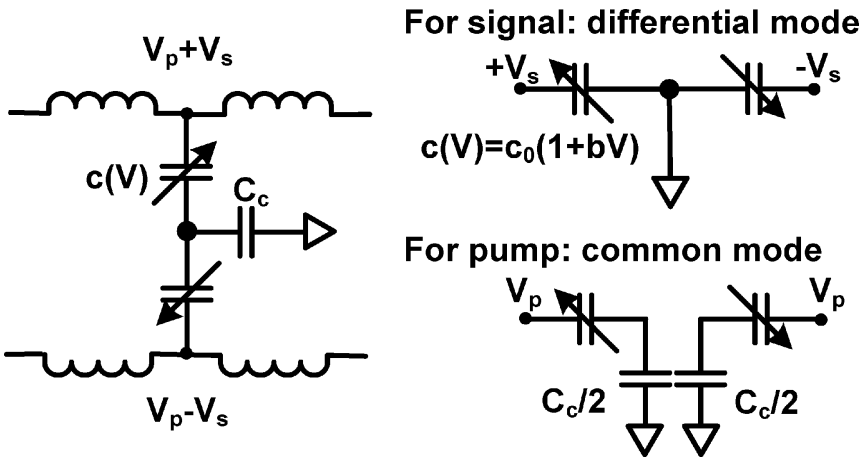

Fig. 4. Proposed phase-matched line: $C_{c}$ compensates for the decrease in the pump propagation velocity due to the dispersion.

$\beta_{p}$, was set to be exactly twice the signal propagation constant, $\beta$. In other words, the signal and the pump were assumed to co-propagate at the same velocity, or be synchronized for maximum energy transfer from the pump to the signal. However, in a transmission line that has a cutoff frequency comparable with the pump frequency for an optimized gain, the dispersion is not negligible. In this case, the deviation from $\beta_{p}=2 \beta$ results in a gain drop. It can be shown that, with the phase mismatch of $\Delta \beta=\beta_{p}-2 \beta$, the exponential coefficient becomes [9]

$$
s=\sqrt{\left(\frac{\beta b A_{p}}{4}\right)^{2}-\left(\frac{\Delta \beta}{2}\right)^{2}} .
$$

From (16), we can see that parametric amplification does not occur when $\Delta \beta / \beta$ is larger than $b A_{p} / 2$. Inserting the typical values for $b=0.5 \mathrm{~V}^{-1}$ and $A_{p}=0.5 \mathrm{~V}$, the allowable $\Delta \beta / \beta$ is $12.5 \%$ for a nonzero parametric gain. Considering the transmission-line loss caused by a limited quality factor of each element, the range becomes even narrower.

The most critical point is that a small phase mismatch between signal and pump is accumulated as the signal propagates over multiple sections. If the accumulated phase mismatch reaches a certain point, further propagation gives attenuation instead of amplification following the expression of $V(x, t)$ in (14). The allowable accumulated phase mismatch is between $\pi / 4$ and $\pi / 2$, depending on the system parameters such as pump amplitude and varactor nonlinearity.

In optics, a method of quasi-phase matching is proposed to resolve this problem by changing the degree of the nonlinearity periodically over the propagation distance [19]. Here, we propose a simpler solution based on two parallel nonlinear transmission lines with "dispersion compensation capacitors" as shown in Fig. 4. In this scheme, the signal is applied differentially to two parallel lines while the pump is a common-mode signal. For differential signals, the net average capacitance is $C_{0}$ due to the virtual ground. However, the net average capacitance for the pump drops to $C_{0}$ in series with $\left(C_{c} / 2\right)$ due to the dispersion compensation capacitor. This decreases the pump propagation delay without changing the signal propagation, cancelling the dispersion of the nonlinear transmission line. Fig. 5 shows the effect of proposed phase matching on parametric amplification using Spectre in Cadence. 


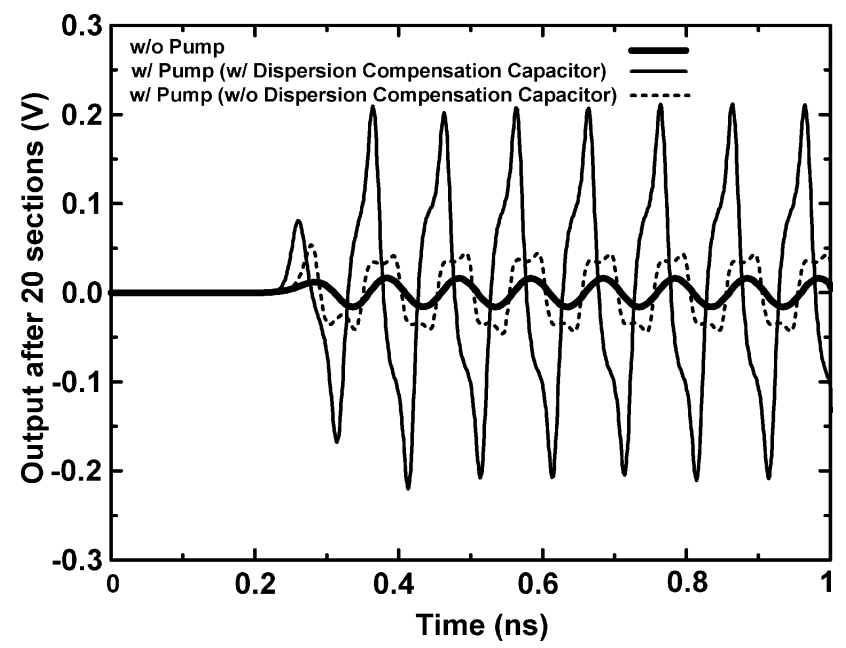

Fig. 5. Effect of the dispersion compensation capacitor $C_{c}$ for phase matching $\left(\omega=10 \mathrm{GHz}, n=20, l=630 \mathrm{pH}, c_{0}=250 \mathrm{fF}, C_{C}=1.2 \mathrm{pF}\right)$.

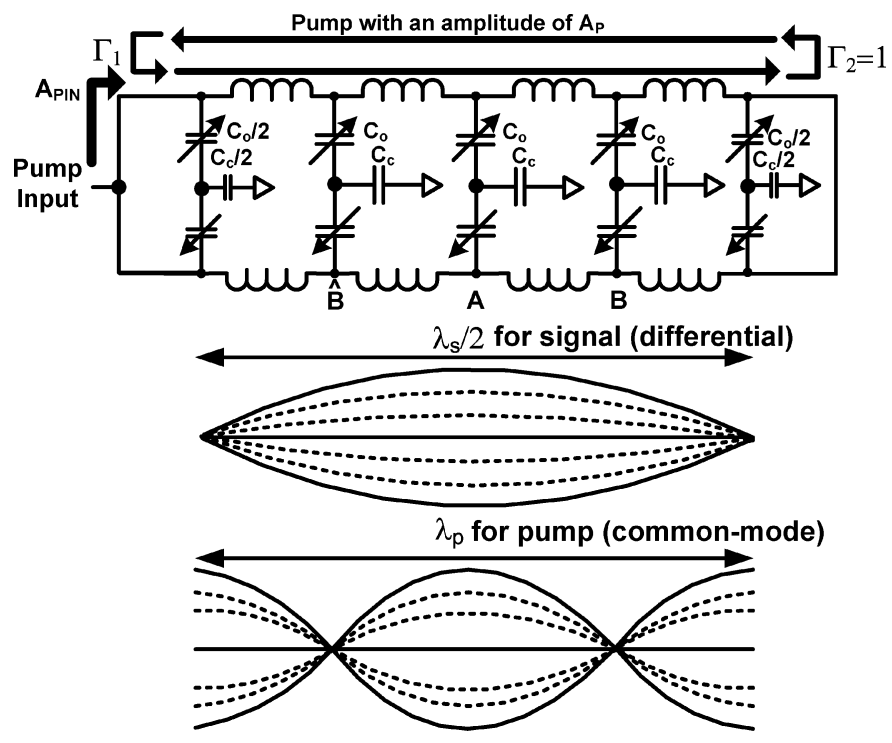

Fig. 6. Distributed resonator with two reflective ends and different standing wave formation for pump and signal.

\section{STANDING-WAVE PARAMETRIC OSCILlATOR: A FREQUENCY DIVIDER}

Parametric oscillation occurs when the parametric amplification compensates the loss of a resonator at the resonance frequency. Here, we use this concept to demonstrate a frequency divider by considering the pump as the input signal. Fig. 6 shows the structure of the proposed parametric oscillator. It consists of two transmission lines with four phase-matched $L C$ sections that are tied at both ends. Oscillation starts from the ambient thermal noise of the resonator when the pump is strong enough to compensate the loss. Any arbitrary thermal noise pair on the differential transmission lines can be represented as the sum of common-mode and differential components. However, only differential component grows by traveling back and forth between two reflective ends through the degenerate parametric amplification since the phase match is achieved as shown in Fig. 4. On the other hand, the common-mode component is suppressed due to the resonator loss. The amplitude of the differential component will increase up to the point where the gain and the loss are equal due to the gain saturation. The gain saturation occurs through pump depletion which means that the pump level is depleted due to the large energy flow from the pump to the signal frequency.

In order to calculate the steady-state output amplitude, we start with the coupled-mode equations (10) and (11) in Section II for $\phi=\pi / 2$. After adding the transmission-line loss and the pump depletion in these equations, the coupled-mode equations can be modified to

$$
\begin{aligned}
\frac{\partial A}{\partial x} & =-\alpha_{s} A+\frac{\beta b A_{p}}{4} A^{*} \\
\frac{\partial A^{*}}{\partial x} & =-\alpha_{s} A^{*}+\frac{\beta b A_{p}}{4} A \\
\frac{\partial A_{p}}{\partial x} & =-\alpha_{p} A_{p}-\beta b|A|^{2}+\kappa A_{\text {pin }}
\end{aligned}
$$

where $\alpha_{s}$ and $\alpha_{p}$ are the attenuation constants of the transmission line at signal and pump frequencies, respectively, $A_{\text {pin }}$ is the input amplitude into each transmission line of the resonator, and $\kappa A_{\text {pin }}$ represents the increase in the pump amplitude inside the resonator due to the input signal. The significance of this term will be seen for the standing wave resonator when reflections are taken into account, which will be presented in (26). In (19), the second term on the right corresponds to the pump depletion. This term can be easily obtained by adding the second-harmonic generation of the signal in (4) to (5).

For the steady-state solution where gain and loss are equal, so that the signal amplitude is constant over the propagation, $\partial A / \partial x, \partial A^{*} / \partial x, \partial A_{p} / \partial x$ are set to be zero. In this case, combining (17) and (18) results in

$$
|A|_{\text {steady }}=0 \text { or }\left.\quad A_{p}\right|_{\text {steady }}=\frac{4 \alpha_{s}}{\beta b} .
$$

Nonzero output condition leads us to choose $\left.A_{p}\right|_{\text {steady }}=$ $4 \alpha_{s} / \beta b$ in (20) and to insert this into (19), which can be rearranged as ${ }^{1}$

$$
\begin{aligned}
|A|_{\text {steady }}^{2} & =\frac{1}{\beta b}\left(\kappa A_{\text {pin }}-\left.\alpha_{p} A_{p}\right|_{\text {steady }}\right) \\
& =\left.\frac{\kappa^{2}}{4 \alpha_{s} \alpha_{p}} A_{\text {pin }}\right|_{\text {th }} ^{2}\left(\frac{A_{\text {pin }}}{\left.A_{\text {pin }}\right|_{\mathrm{th}}}-1\right) \text { for } A_{\text {pin }}>\left.A_{\text {pin }}\right|_{\text {th }}
\end{aligned}
$$

where a threshold input pump amplitude is given by

$$
\left.A_{\text {pin }}\right|_{t h}=\left.\frac{\alpha_{p}}{\kappa} A_{p}\right|_{\text {steady }} \text {. }
$$

At the resonator end where the pump is injected, the relation between $m$ th and $(m+1)$ th round-tripped pump amplitudes, $A_{p_{m}}$ and $A_{p_{m+1}}$, is

$$
A_{p_{m+1}}=A_{p_{m}} \exp \left(-2 \alpha_{p} d\right)+A_{\text {pin }}
$$

where $2 d$ is the round-trip length of the resonator. In the steady state, $A_{p_{m+1}}=A_{p_{m}}=\left.A_{p}\right|_{\text {steady }},\left.A_{p}\right|_{\text {steady }}$ linearly increases with $A_{\text {pin }}$ for $A_{\text {pin }} \leq\left. A_{\text {pin }}\right|_{\text {th }}$ by inserting $|A|_{\text {steady }}=0$ into (19). However, $\left.A_{p}\right|_{\text {steady }}$ becomes constant with $A_{\text {pin }}$ once a

\footnotetext{
${ }^{1}$ The steady-state solution turns out to be unconditionally stable from nonlinear stability theory.
} 


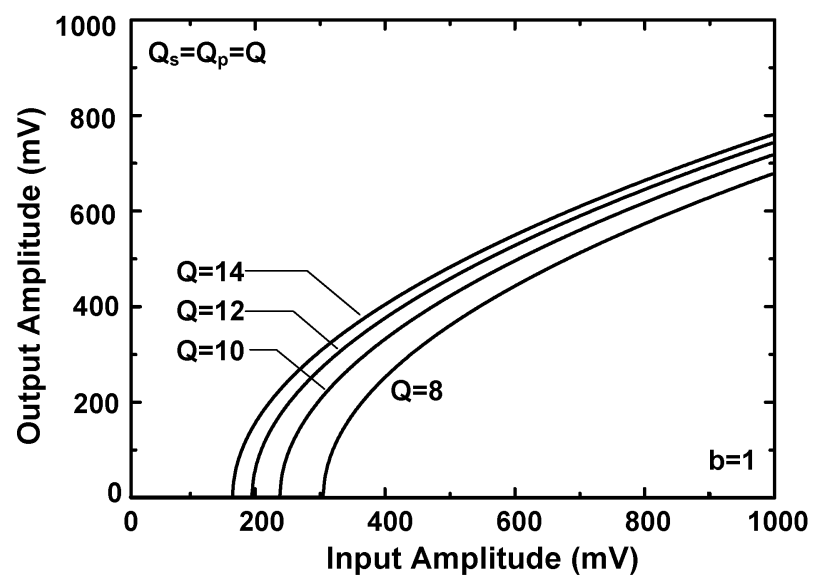

(a)

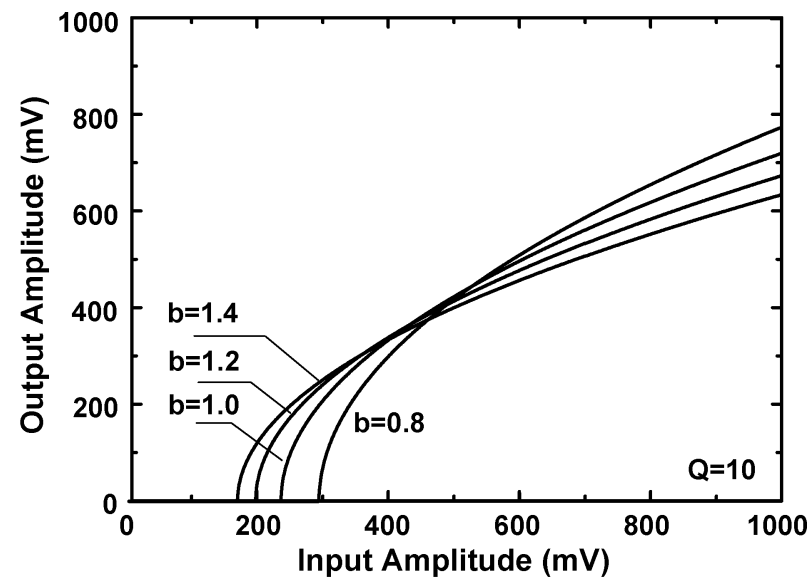

(b)

Fig. 7. Calculated output amplitude versus input amplitude. (a) Effect of loss represented by different quality factors at $10 \mathrm{GHz}$. (b) Effect of nonlinearity represented by $\mathrm{C} / \mathrm{V}$ slope $b$. $\left(f_{c}=25 \mathrm{GHz}, f_{\text {in }}=20 \mathrm{GHz}, Z_{o}=50 \Omega, Z_{\text {out }}=50 \Omega, n=4\right)$.

parametric oscillation starts for $A_{\text {pin }}>\left.A_{\text {pin }}\right|_{\text {th }}$. Hence, beyond an oscillation threshold, (24) becomes

$$
\left.A_{\text {pin }}\right|_{t h}=\left.A_{p}\right|_{\text {steady }}\left(1-\exp \left(-2 \alpha_{p} d\right)\right) .
$$

Combining (23) and (25), $\kappa$ is reduced to

$$
\kappa=\frac{\alpha_{p}}{\left(1-\exp \left(-2 \alpha_{p} d\right)\right)} .
$$

From (22) and (26), the steady-state amplitude of the output signal becomes

$$
\begin{aligned}
& |A|_{\text {steady }}=\frac{\left.A_{\text {pin }}\right|_{t h}}{2\left(1-\exp \left(-2 \alpha_{p} d\right)\right)} \sqrt{\frac{\alpha_{p}}{\alpha_{s}}} \sqrt{\frac{A_{\text {pin }}}{\left.A_{\text {pin }}\right|_{t h}}-1} \\
& \text { where }_{\left.A_{\text {pin }}\right|_{\text {th }}}=\frac{4 \alpha_{s}\left(1-\exp \left(-2 \alpha_{p} d\right)\right)}{\beta b}=\frac{2\left(1-\exp \left(-2 \alpha_{p} d\right)\right)}{Q_{s} b} .
\end{aligned}
$$

Note that $|A|_{\text {steady }}$ is a steady-state amplitude of a traveling wave in one direction and that the measured voltage at output node is the superposition of forward and backward waves, forming a standing wave. Therefore, the output voltage measured at $x_{\text {out }}$ is $\gamma|A|_{\text {steady }}$ where $\gamma \simeq 2\left|\sin \left(\beta x_{\text {out }}\right)\right|$ for a resonator length of $d=\lambda_{s} / 2$.

So far, we assumed that the pump is perfectly reflected back at both ends of the resonator. However, we connect the external signal source with a finite output impedance, $Z_{\text {out }}$, at one end of the resonator to supply a pump. This finite output impedance makes the input-side reflection coefficient, $\Gamma_{1}$, smaller than 1 , thereby resulting in additional loss of pump. As a result, we need to introduce an effective pump loss $\alpha_{\text {pe }}$ to replace $\alpha_{p}$ which solely comes from the transmission-line loss. The pump roundtrip loss including the additional loss due to imperfect reflection can be expressed by

$$
\begin{aligned}
\exp \left(-2 \alpha_{p e} d\right) & =\Gamma_{1} \exp \left(-2 \alpha_{p} d\right) \\
\alpha_{p e} & =\alpha_{p}-\frac{\ln \Gamma_{1}}{2 d}
\end{aligned}
$$

where $\Gamma_{1}$ is the input-side reflection coefficient given by

$$
\Gamma_{1}=\frac{2 Z_{\text {out }}-Z_{0}}{2 Z_{\text {out }}+Z_{0}}
$$

and $Z_{0}$ is the characteristic impedance of each transmission line. It is noted that the differential oscillation signal does not see the signal source impedance due to the virtual ground.

Fig. 7 shows the calculated output amplitude versus the input amplitude based on (27) after replacing $\alpha_{p}$ with $\alpha_{p e}$. To investigate the effect of the loss and the nonlinearity, we change the quality factor of the transmission line and varactor $\mathrm{C} / \mathrm{V}$ slope $b$. As expected from (28), the oscillation threshold decreases as the resonator loss decreases and the nonlinearity of the varactor increases. However, the slope of the output amplitude versus input amplitude varies in a different way: while lower loss still gives higher slope, the high nonlinearity results in lower slope due to the higher pump depletion. In a real system, additional losses from vias and interconnects decrease the effective quality factor of the line, and the parasitic capacitance around transmission lines decreases the nonlinearity. These nonideal effects require careful attention to layout.

The lower part of Fig. 6 shows different standing-wave formations inside the resonator for pump and signal. The differential signal sees the resonator ends as shorts due to the virtual ground, while the common-mode pump sees the ends as open nodes. With the reflection at both ends, the signal forms a $\lambda_{s} / 2$ standing wave with minimum amplitudes at both ends. On the other hand, the pump forms a $\lambda_{p}$ standing wave with maximum amplitudes at both ends. The higher modes for pump and signal are suppressed by the cutoff frequency of the resonator.

The oscillation frequency is determined by the standing-wave formula

$$
d=n h=\frac{\lambda_{s}}{2}=\frac{\pi}{\beta}=\frac{1}{2 f_{\mathrm{osc}} \sqrt{\left(\frac{l}{h}\right)\left(\frac{c_{0}}{h}\right)}}
$$

where $d$ is the physical length of the transmission line, $h$ is the spacing between two adjacent nodes, and $n$ is the number of sections of the resonator. The varactor capacitance per unit section is approximated by its average value, $c_{0}$. Equation (32) can be rearranged to

$$
f_{\mathrm{osc}}=\frac{1}{2 n \sqrt{l c_{0}}}
$$


The loop gain at a given oscillation frequency in a resonator with $n$ sections is roughly

$$
G_{\text {loop }}=\exp \left(\frac{b A_{p} \omega_{\mathrm{osc}} \sqrt{l c_{0}}}{4} \cdot 2 n\right)=\exp \left(\frac{\pi b A_{p}}{2}\right) \text {. }
$$

This equation shows that the loop gain at a fixed oscillation frequency does not depend on the number of sections. In our design, we set $n$ to be four for a small footprint and reasonable attenuation especially for the pump signal. Another reason for having four sections is that we can exploit the different standingwave formations for pump and signal to suppress the pump frequency at the output.

There are three possible nodes for taking the signal out of the resonator as shown in Fig. 6 . A provides the maximum amplitude for both pump and signal, and the pump signal will be filtered out only if the output signal is taken differentially from the two transmission lines. However, we need an extra low-pass filter for a single-ended output at $A$. On the other hand, $B$ or $\hat{B}$ can suppress the pump even for the single-ended output thanks to the standing-wave formation. The down side is a lower signal amplitude compared with $A$. $B$ is more appropriate for the suppression of the pump than $\hat{B}$, because the amplitude imbalance between forward and backward waves due to the loss is smaller in this node. In addition, the external connection for the output adds a parasitic conductance to the resonator, resulting in extra loss. The power dissipation caused by the additional conductance is $P_{\text {diss }}=1 / 2 G V_{p}^{2}$, which is proportional to the square of the pump amplitude. Therefore, connecting the output port at $A$ leads to the maximum loss in the pump amplitude, and it raises the threshold voltage and decreases the amplitude of the signal.

The remaining challenge is how to inject pump power into the resonator effectively. For pump, the resonator is simply two parallel open-ended transmission lines with a length of $\lambda_{p}$. The input impedance looking into one end of the resonator is [20]

$$
\begin{aligned}
Z_{\text {in }} & =\frac{Z_{0}}{2} \frac{1}{\tanh \left(\alpha_{p}+j \beta_{p}\right) \lambda_{p}} \\
& \simeq \frac{Z_{0}}{2}\left[\frac{\alpha_{p} \lambda_{p}-j \cos \left(\beta_{p} \lambda_{p}\right) \sin \left(\beta_{p} \lambda_{p}\right)}{\left(\alpha_{p} \lambda_{p} \cos \left(\beta_{p} \lambda_{p}\right)\right)^{2}+\sin ^{2}\left(\beta_{p} \lambda_{p}\right)}\right]_{\omega=\omega_{p}} \\
& =\frac{Z_{0}}{2 \alpha_{p} \lambda_{p}}=\frac{Q_{p} Z_{0}}{2 \pi}
\end{aligned}
$$

where $Z_{0}$ is the characteristic impedance of each transmission line and $Q_{p}$ is the resonator quality factor at the pump frequency. The input impedance becomes purely real at the resonance frequency and its harmonics. By selecting the right value for the characteristic impedance for a given quality factor, one can achieve the input matching without an additional matching network. Considering that $Q_{p}$ in our design is around 7 in a $0.13-\mu \mathrm{m}$ CMOS process, $Z_{0}$ of $50 \Omega$ provides a good input match to a $50-\Omega$ source according to (37).

\section{Comments on Phase-Noise Performance}

One of the main advantages of our design is a better phasenoise performance than the conventional injection-locked frequency dividers. In an $L C$ oscillator where transistors are used for sustaining oscillation, the channel noise makes the main contribution to the phase noise [22]. Although the phase noise of the injection-locked frequency divider tracks that of the injected

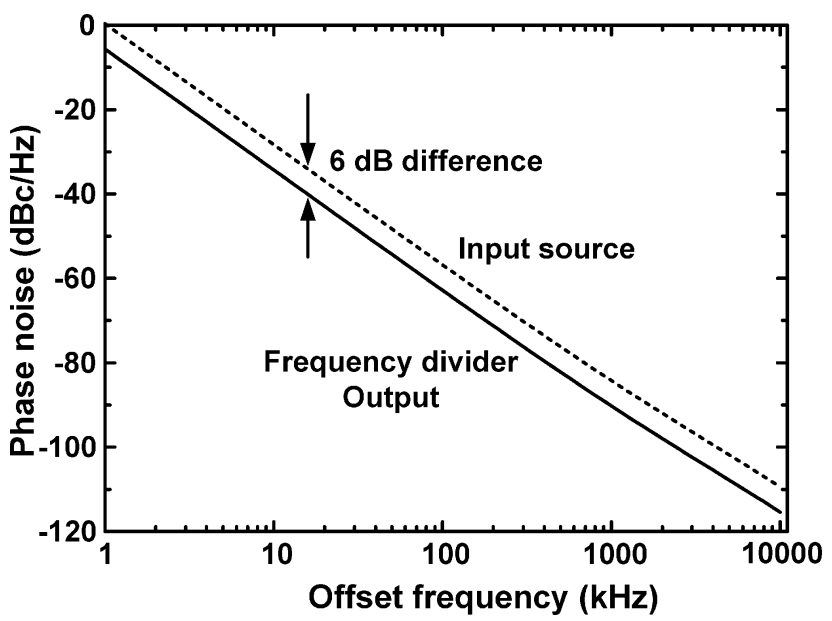

Fig. 8. Simulated phase noise for an input frequency of $20 \mathrm{GHz}$ using Spectre.

signal at low offset frequencies, the excess noise from the divider degrades the phase noise back to the level of a free-running $L C$ oscillator at higher offset frequencies [6]. Our design does not have any transistors, thus guaranteeing that it has a low phase-noise floor [23].

The other interesting point is that our frequency divider has high spectral purity because of its phase-selection property, as shown in (14). Start-up noise such as a thermal noise grows by the degenerate parametric amplification for a pump power over the threshold. The degenerate parametric amplification picks a limited noise component to be amplified in terms of phase and frequency. This is because the degenerate parametric amplification works only for $\omega=\omega_{p} / 2$ and a specific initial phase difference between signal and pump as we discussed in Section II.

Fig. 8 shows the simulated phase-noise performance using Spectre in a $0.13-\mu \mathrm{m}$ CMOS process. The phase-noise difference between input and output is $6 \mathrm{~dB}$ over simulation frequencies, which agrees with an ideal value, $20 \log _{10} N \mathrm{~dB}$, for a divide-by- $\mathrm{N}$ frequency divider [24].

\section{Design And Measurement}

We design a $20-\mathrm{GHz}$ frequency divider based on the parametric oscillator in a $0.13-\mu \mathrm{m}$ CMOS process. First, we set the cutoff frequency of the resonator for maximum gain as discussed in Section II. In our design, this optimum value is 25 $\mathrm{GHz}$ that determines the product of the inductance and capacitance. The number of sections is set to four as discussed before. These values for the section number and the cutoff frequency guarantee a resonant frequency of $10 \mathrm{GHz}$. Then, the dispersion compensation capacitor is determined by phase-matching condition between the pump and the signal. The phase matching can be verified by making sure that the resonance frequency of $S_{11}$ is exactly at $20 \mathrm{GHz}$. Without the dispersion compensation, $S_{11}$ has a resonance frequency lower than $20 \mathrm{GHz}$. Finally, depending on the magnitude of the input matching, the characteristic impedance can be selected which gives the ratio of inductance and capacitance. A buffer is added to the output node as shown in Fig. 9. Note that the buffer is only needed for testing purposes using a $50-\Omega$ load. The $C_{\mathrm{gs}}$ of the buffer transistor adds parasitic capacitance to the nonlinear transmission line, thereby decreasing the nonlinearity of an $L C$ section where the output 


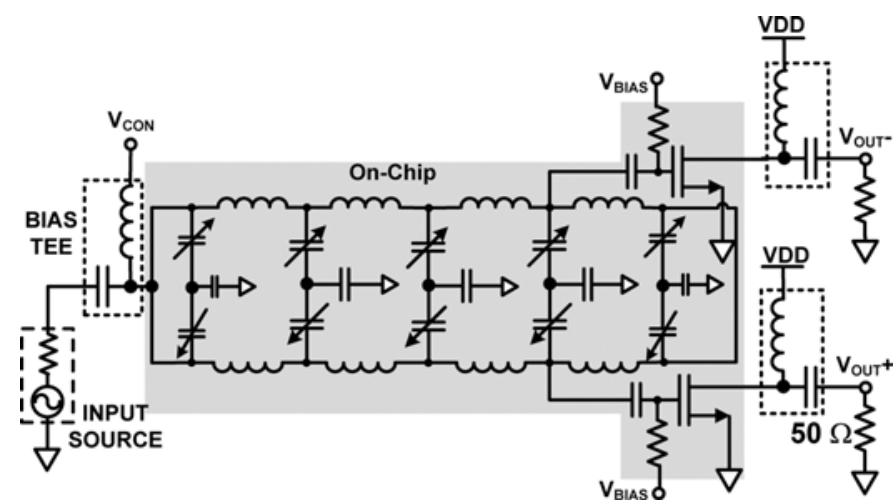

Fig. 9. Schematic of the frequency divider with buffer and control voltage for frequency tuning.

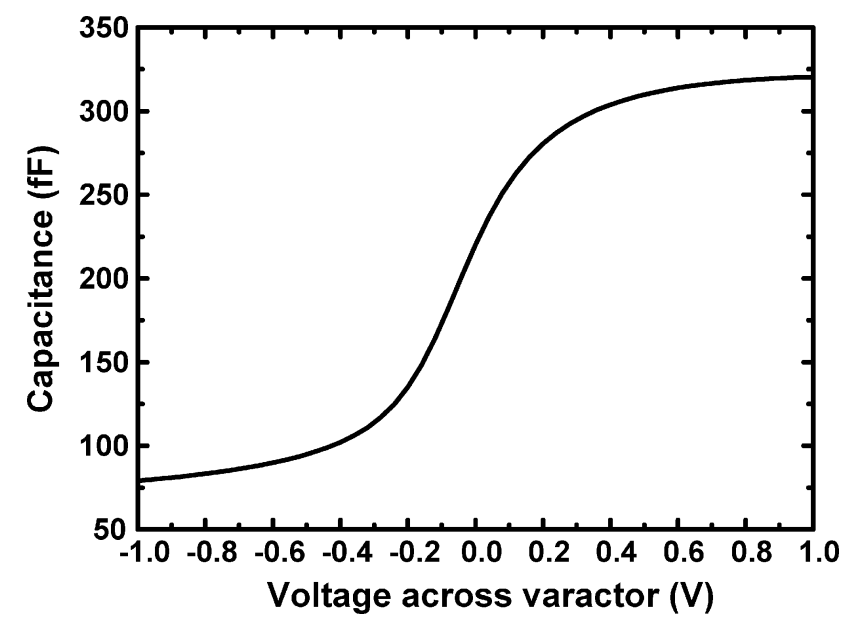

Fig. 10. Varactor capacitance versus bias voltage in a $0.13-\mu \mathrm{m}$ process.

node is placed. However, the use of multiple $L C$ sections alleviates this effect. Moreover, the buffer capacitance does not affect the operation frequency since it is absorbed into the transmission line. There are two output ports, which give us the option to have single-ended or differential outputs. The oscillation frequency can be tuned by controlling $V_{\mathrm{CON}}$ through the bias tee at the input port.

We use ground-shielded spiral inductors with quality factors of around 20 at $20 \mathrm{GHz}$. In this design, the inductors are around $510 \mathrm{pH}$ and occupy $100 \mu \mathrm{m} \times 100 \mu \mathrm{m}$. Accumulation-mode nMOS varactors are used as voltage-dependent capacitors. The capacitance versus voltage of these capacitors is shown in Fig. 10. The ratio of minimum-to-maximum capacitances is around 3.5, which represents the nonlinearity of the system. The varactors use the multifinger gate structure to optimize the nonlinearity and the quality factor simultaneously [25]. For dispersion compensation capacitors, vertical natural capacitors (VNCAPs) are used due to their high quality factor [18].

Fig. 11 shows the chip photograph implemented in a $0.13-\mu \mathrm{m}$ CMOS process. The core area of the die is $0.75 \times 0.32 \mathrm{~mm}^{2}$, not including pads. As the design frequency increases, the sizes of the inductors are scaled down and the whole structure can be made of two parallel transmission lines without spiral inductors. In other words, this frequency divider can easily fit in the entire system layout with great flexibility.

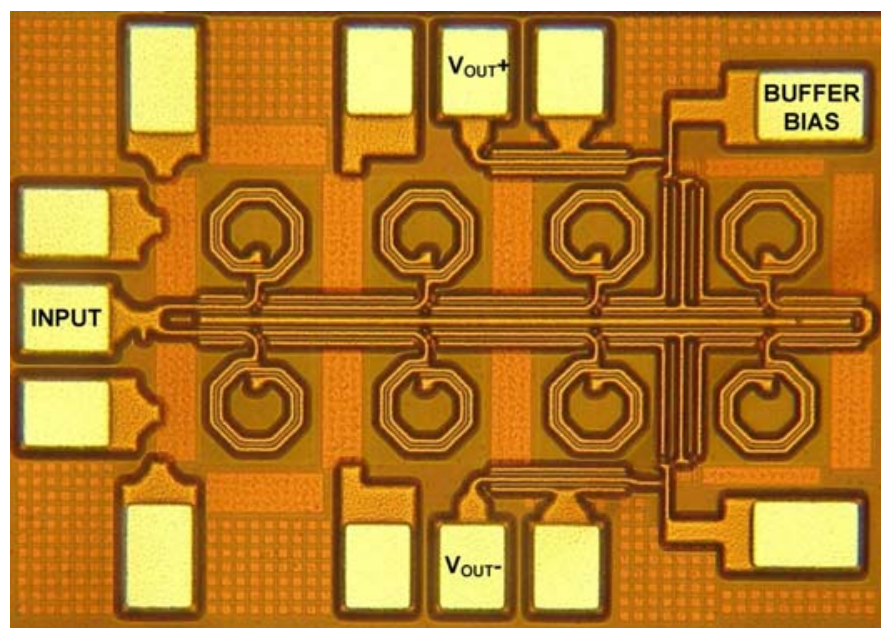

Fig. 11. Die photograph of the chip.

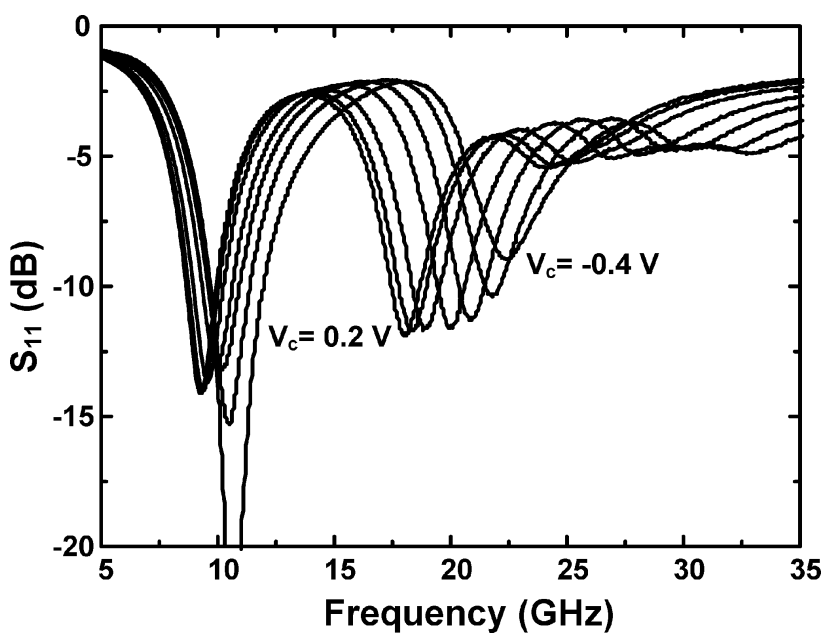

Fig. 12. Measured input matching for different control voltages from -0.4 to $0.2 \mathrm{~V}$.

The frequency divider is measured using an Agilent E8257D signal generator for the pump signal and an Agilent 8564EC spectrum analyzer. To measure the input matching, an Agilent E8364B network analyzer is used. We de-embed the loss of all cables, adapters, bias tees, and probes for a more accurate measurement. The bias current for the buffer is set to be $8 \mathrm{~mA}$ from a $1.2-\mathrm{V}$ power supply.

$S_{11}$ is measured to investigate the input matching while changing the control voltage. As shown in Fig. 12, our frequency divider has a reasonable matching performance of $S_{11}<-10 \mathrm{~dB}$ over $18-22 \mathrm{GHz}$.

Next, the single-ended output spectrum is measured against the input (pump) amplitude as shown in Fig. 13. For an input amplitude below the threshold, the output spectrum only shows the input frequency, $f_{\text {in }}$. However, as the input amplitude increases beyond the threshold, the $f_{\text {in }} / 2$ component appears and grows with the input amplitude. Note that the output spectrum at the input frequency stays almost constant as the input amplitude increases, which means that most of the input energy over the threshold is transferred to the $f_{\text {in }} / 2$ component. This can be also predicted by (20). In addition, the proposed standing wave formation leads to the suppression of the input frequency at the 


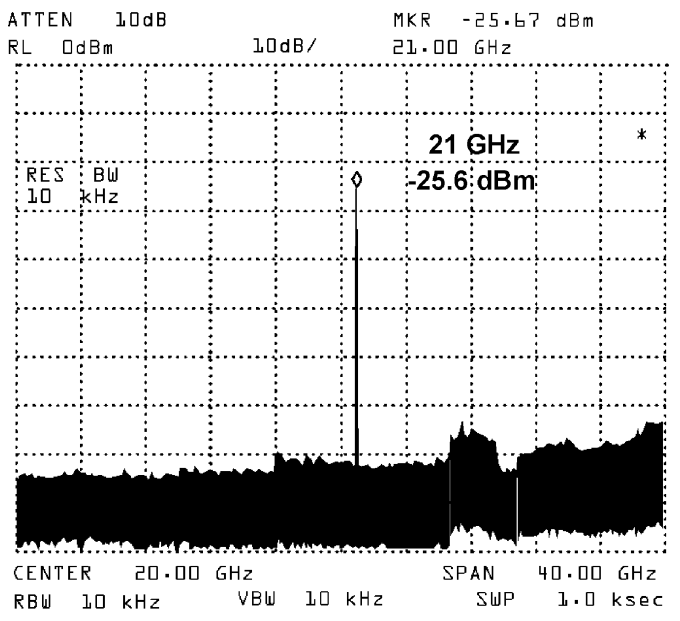

(a)

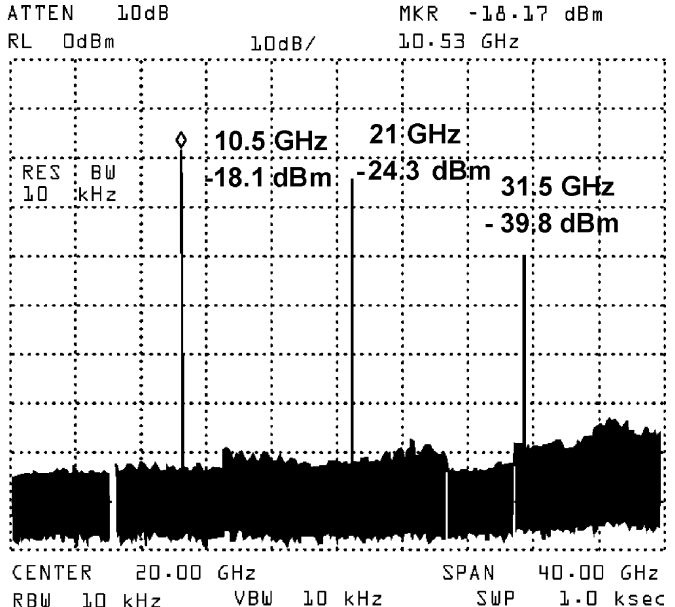

(b)

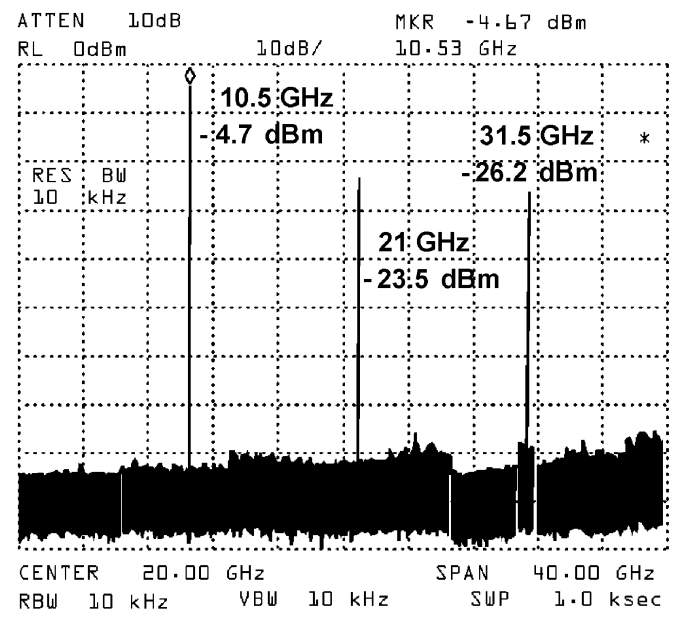

(c)

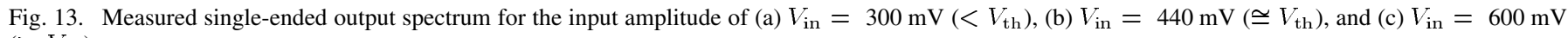
$\left(>V_{\mathrm{th}}\right)$.

single-ended output. A $3 f_{\text {in }} / 2$ component is also generated by nonlinear coupling between $f_{\text {in }} / 2$ and $f_{\text {in }}$ components. The harmonics higher than $3 f_{\text {in }} / 2$ are negligible due to the resonator cutoff frequency.

The output amplitude versus the input amplitude is simulated and measured in Fig. 14. The measured input threshold is around $400 \mathrm{mV}$, while the simulated value is $300 \mathrm{mV}$. The slope of the output amplitude versus input amplitude in the measurement is higher than that of the simulation. This difference can be explained by Fig. 7(b). The drop in the nonlinearity caused by the parasitic capacitances (which are linear) increases both the threshold and the slope simultaneously, while some additional loss also contributes to the increase in the threshold. The output amplitude increases with the input amplitude over the threshold following a square-root relation as explained in (27). The $600-\mathrm{mV}$ input amplitude results in a $300-\mathrm{mV}$ singleended output amplitude (600-mV differential output) due to its high-frequency conversion efficiency as well as the standing wave formation. The calculation result is also plotted in Fig. 14 to verify the analysis in Section III. All of the parameters for calculation, including quality factors and varactor nonlinearity, are extracted from the Cadence simulation and the simulated

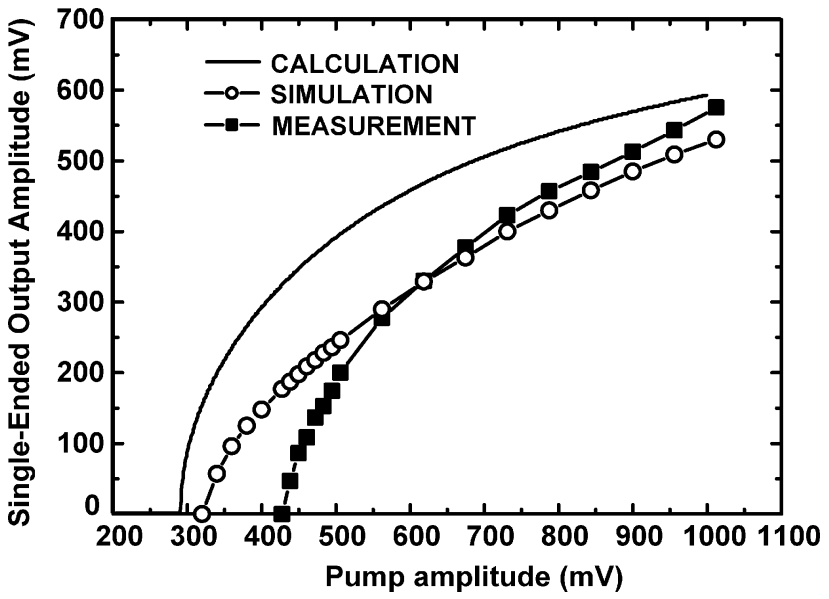

Fig. 14. Output amplitude at $f_{\text {in }} / 2$ versus input amplitude at $f_{\text {in }}$.

transfer function of the output buffer is included for accurate comparison.

The efficiency of the frequency conversion is simulated in terms of pump depletion ratio, which is defined as the ratio of the 


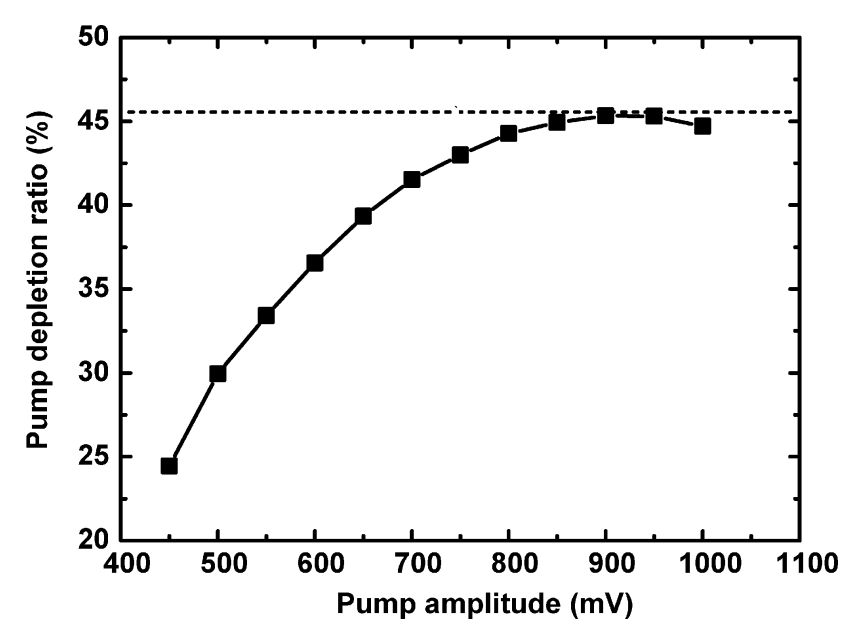

Fig. 15. Simulated pump depletion ratio for investigation of the frequency conversion efficiency.

drop in the pump power to the injected pump power after parametric oscillation. Pump depletion occurs because parametric oscillation results in power transfer from the pump to the signal. As the power is transferred, the level of the pump amplitude on the resonator decreases. Therefore, the higher pump depletion means more efficient frequency conversion. However, there are some backconversion processes which drop the efficiency. The sufficiently grown signal at $f_{\text {in }} / 2$ generates the second harmonic at $f_{\text {in }}$, which returns the power back to the pump. Besides the backconversion by the second harmonic generation, the interaction between signal and pump generates $3 f_{\text {in }} / 2$ component. Then, the interaction between $3 f_{\text {in }} / 2$ and $f_{\text {in }} / 2$ components increases the $f_{\text {in }}$ component through nondegenerate parametric amplification [21]. In Cadence simulation, the pump depletion is found to be $45 \%$ as shown in Fig. 15. To exclude the nonlinearity of the buffer, the pump amplitude is simulated before the buffer. The harmonic suppression ratio at the singleended output is also measured as shown in Fig. 16. The suppression ratio for the second $\left(f_{\text {in }}\right)$ and third $\left(3 f_{\text {in }} / 2\right)$ harmonics are more than $16 \mathrm{~dB}$ due to the standing-wave formation and the resonator cutoff frequency that is lower than $3 f_{\text {in }} / 2$. Transient signals are also measured for single-ended and differential outputs using an Agilent 86100C digital communication analyzer with $80-\mathrm{GHz}$ bandwidth as shown in Fig. 17.

We measure the threshold voltages over input frequencies for different control voltages to find the tunability as shown in Fig. 18. This result demonstrates a wide tuning range from 18.5 to $23.5 \mathrm{GHz}(24 \%)$. As the control voltage departs from zero, the $\mathrm{C} / \mathrm{V}$ slope of the varactor reduces due to the change of the varactor operation regime as shown in Fig. 10. As a result, the effective nonlinearity decreases, thereby increasing the threshold voltage. In addition, the dispersion compensation capacitor is still fixed with the change of the varactor, resulting in some phase mismatch. This is another reason for the increased threshold voltage. The increased threshold voltage might limit the use of the proposed frequency divider for low power applications. However, this can be addressed by optimizing the resonator structure and/or increasing the nonlinearity of the varactor: a standing-wave formation can be exploited to maximize the quality factor of the resonator by spatially manipulating the

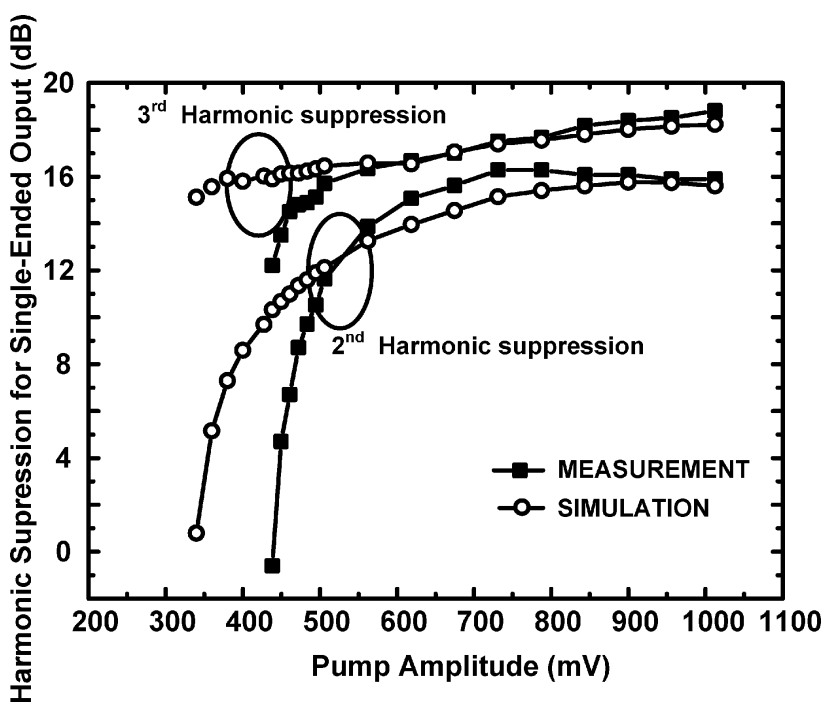

Fig. 16. Measured second and third harmonic suppression ratio at the singleended output.

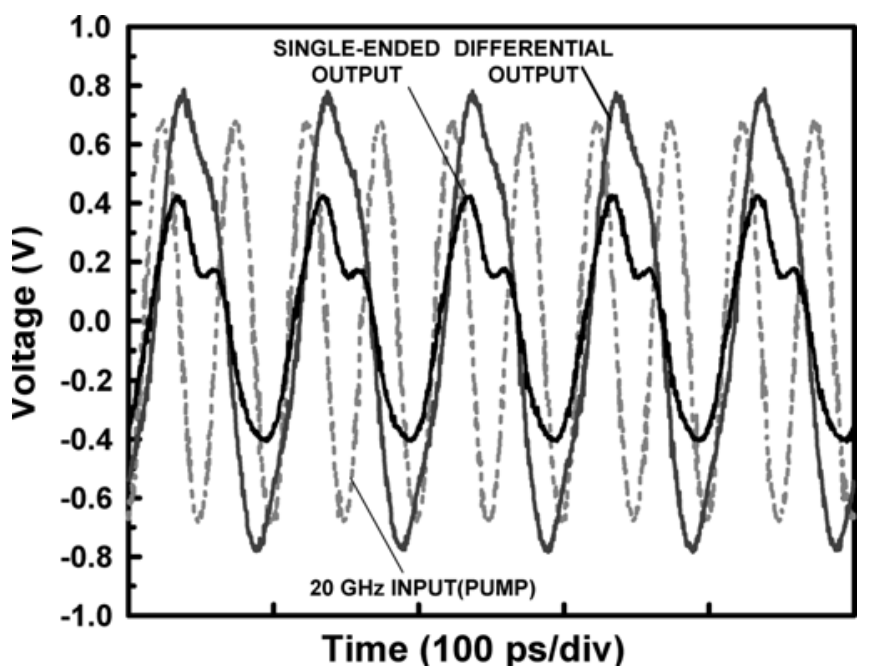

Fig. 17. Measured output waveform for a $20 \mathrm{GHz}$ input.

characteristic impedance [26]. In addition, we can increase the varactor nonlinearity by increasing $C_{\text {ox }}$ since the varactor capacitance is the series combination of the $C_{\mathrm{ox}}$ and voltage-dependent channel capacitance[27]. The CMOS process scaling can result in this desired increase.

For a fixed varactor bias, the operation bandwidth is relatively small, which is another limiting factor of the proposed frequency divider. Basically, the proposed structure is a wavebased oscillator and the round-trip phase is equal to $2 \pi$ at the oscillation frequency which is half of the pump frequency. When this frequency is the resonant frequency of the structure, the oscillation signal and its nonlinear interaction term with the pump should have the same phase to maintain the round-trip phase of $2 \pi$. This leads to $\phi=\pi / 2$ in (14), which gives the maximum parametric gain. However, when the divide-by-two frequency is deviated from the resonant frequency, the pump selects a signal phase for oscillation that is different from the nonlinear interaction term. The vector sum of the signal and the nonlinear interaction term results in a certain phase shift to satisfy the round-trip 


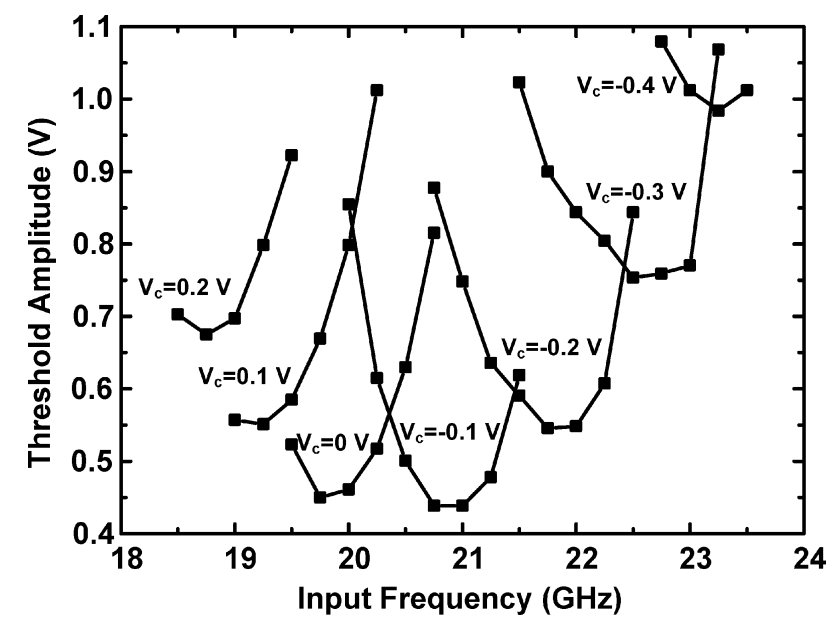

Fig. 18. Measured threshold versus input frequency for different control voltages.

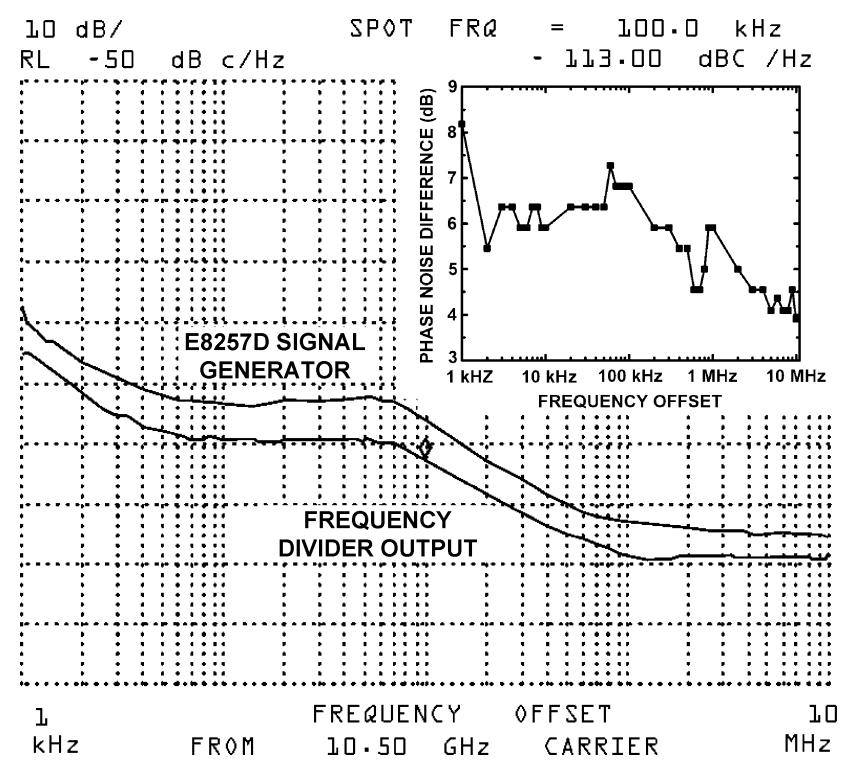

Fig. 19. Measured phase noise for signal generator and frequency-divider output.

phase condition. The deviation from $\phi=\pi / 2$ leads to lower parametric gain, thereby increasing the threshold.

Fig. 19 shows the measured phase noise. The phase noise difference between input and output is around $6 \mathrm{~dB}$ over a wide range of the offset frequency, which agrees with the simulation result shown in Fig. 8. Above 1-MHz offset frequency, the noise floor of the spectrum analyzer becomes dominant and the measured phase noise difference decreases.

\section{DISCUSSION}

Here, we provide comparisons between the proposed frequency divider and a digital frequency divider with no static power consumption in terms of the operation speed. Additionally, we discuss the application of the proposed frequency divider in a PLL and its advantages over other types of the frequency dividers.

\section{A. Comparison With Digital Frequency Dividers}

Digital frequency dividers can be also implemented using dynamic latches to achieve no static power consumption. However, the dynamic latch has a long time constant due to the switching resistance, $1 / g_{\mathrm{ds}}$, and parasitic capacitance of the MOSFET, requiring a rail-to-rail input to minimize the resistance [29]. This is why current-mode logic (CML) latches are commonly used for high-frequency frequency division above $10 \mathrm{GHz}$ despite their high power consumption [30]. Therefore, the proposed frequency divider can operate at higher frequencies than a dynamic latch frequency divider although both of them have no static power consumption. In addition, the fan-out capacitance, which comes from the buffer or the next stage, can slow down the digital frequency divider [29]. On the other hand, the proposed structure can absorb the fan-out capacitances to the transmission line structure to increase the operation frequency. However, the digital frequency divider, which does not use the resonator structure, provides broader operation bandwidth than the proposed structure.

\section{B. Application in a PLL}

Comparing to Miller frequency dividers and injection-locked frequency dividers, which support a high frequency division, the proposed frequency divider has simpler structure with no static power consumption. This is critical since the overall power consumption of a PLL is dominated by the first few dividers above $10 \mathrm{GHz}$ [1]. Moreover, the absence of active devices alleviates the phase noise contribution in a PLL, caused by the flicker noise and white noise floor [31].

The distributed structure of the proposed frequency divider is also advantageous for its use in a PLL to cover a large area of clock distribution network. Wiring differential transmission lines in a clock distribution network can be turned into the proposed frequency divider simply by placing the varactors with appropriate spacing and by connecting both ends. However, the narrow input bandwidth for a fixed varactor bias should be improved for reliable operation while the proposed frequency divider can be still useful as one of the first few frequency dividers in a PLL [32].

\section{CONCLUSION}

We propose the first passive CMOS frequency divider based on the parametric amplification process using a reflective distributed resonator. The input signal injected into the resonator transfers its energy into a divide-by-2 frequency component through degenerate parametric amplification. By traveling back and forth in the resonator, the signal grows and finally achieves the steady-state oscillation at a divide-by-2 frequency. Not having any active devices to sustain oscillation has two important advantages: no static power consumption and low phase noise. Furthermore, the reflective distributed resonator forms different standing waves for signal and pump, which is exploited to suppress the pump at the output as well as to increase the signal amplitude. Finally, the proposed divider occupies more chip area compared to the conventional designs unless it is implemented as part of the clock distribution network. 


\section{ACKNOWLEDGMENT}

The authors would like to thank G. Li, O. Momeni, Y. Tousi, M. Adnan, E. Hwang, and Prof. Apsel of Cornell University, Ithaca, NY, for helpful discussions regarding various aspects of this work, the TSMC University Shuttle Program for chip fabrication, and M. Azarmnia and $\mathrm{H}$. Yu for their support.

\section{REFERENCES}

[1] A. M. Niknejad and H. Hashemi, mm-Wave Silicon Technology: 60 GHz and Beyond. New York: Springer-Verlag, 2008.

[2] S. Rong, A. W. Ng, and H. C. Luong, " $0.9 \mathrm{~mW} 7 \mathrm{GHz}$ and $1.6 \mathrm{~mW} 60$ $\mathrm{GHz}$ frequency dividers with locking-range enhancement in $0.13 \mu \mathrm{m}$ CMOS," in IEEE ISSCC Tech. Dig. Papers, Feb. 2009, pp. 96-97.

[3] K. H. Tsai, L. C. Cho, J. H. Wu, and S. I. Lu, "3.5 mW W-band frequency divider with wide locking range in $90 \mathrm{~nm}$ CMOS technology," in IEEE ISSCC Tech. Dig. Papers, Feb. 2008, pp. 466-467.

[4] Q. Gu et al., "A low power $V$-band CMOS frequency divider with wide locking range and accurate quadrature output phases," IEEE J. Solid-State Circuits, vol. 43, no. 4, pp. 991-998, Apr. 2008.

[5] H. R. Rategh and T. H. Lee, "Superharmonic injection-locked frequency dividers," IEEE J. Solid-State Circuits, vol. 34, no. 6, pp. 813-821, Jun. 1999.

[6] S. Verma, H. R. Rategh, and T. H. Lee, "A unified model for injectionlocked frequency dividers," IEEE J. Solid-State Circuits, vol. 38, no. 6, pp. 1015-1027, Jun. 2003.

[7] B. Razavi, "A study of injection locking and pulling in oscillators," IEEE J. Solid-State Circuits, vol. 39, no. 9, pp. 1415-1424, Sep. 2004.

[8] B. Razavi, "Heterodyne phase locking: A technique for high-speed frequency division," IEEE J. Solid-State Circuits, vol. 42, no. 12, pp. 2887-2892, Dec. 2007.

[9] W. H. Louisell, Coupled Mode and Parametric Electronics. New York: Wiley, 1960.

[10] R. Landauer, "Parametric amplification along nonlinear transmission lines," J. Appl. Phys., vol. 31, pp. 479-484, 1960.

[11] G. R. Sloan, "The modeling, analysis, and design of filter-based parametric frequency dividers," IEEE Trans. Microw. Theory Tech., vol. 55, no. 2, pp. 224-228, Feb. 1993.

[12] A. Suarez and R. Melville, "Simulation-assisted design and analysis of varactor-based frequency multipliers and dividers," IEEE Trans. Microw. Theory Tech., vol. 54, no. 3, pp. 1166-1179, Mar. 2006.

[13] Z. Heshmati, I. C. Hunter, and R. D. Pollard, "Microwave parametric frequency dividers with conversion gain," IEEE Trans. Microw. Theory Tech., vol. 41, no. 10, pp. 2059-2064, Oct. 2007.

[14] A. Yariv and W. H. Louisell, "Theory of the optical parametric oscillator," IEEE J. Quantum Electron., vol. QE-2, no. 9, pp. 418-424, Sep. 1966.

[15] S. E. Harris, "Tunable optical parametric oscillators," Proc. IEEE, vol. 57, no. 12, pp. 2096-2113, Dec. 1969.

[16] P. K. Tien and H. Suhl, "A traveling-wave ferromagnetic amplifier," Proc. IRE, vol. 46, pp. 700-706, Apr. 1958.

[17] P. K. Tien, "Parametric amplification and frequency mixing in propagating circuits," J. Appl. Phys., vol. 29, no. 9, pp. 1347-1357, Sep. 1958.

[18] “CMRF8SF Model Reference Guide," IBM Microelectronics Division, 2007.

[19] L. E. Myers, R. C. Eckardt, M. M. Fejer, and R. L. Byer, "Quasiphase-matched optical parametric oscillators in bulk periodically poled $\mathrm{LiNbO}_{3}$," J. Opt. Soc. Amer. B, vol. 12, no. 11, pp. 2102-2116, Nov. 1995.

[20] D. M. Pozar, Microwave Engineering. New York: Wiley, 2005.

[21] G. M. Roe and M. R. Boyd, "Parametric energy conversion in distributed systems," Proc. IRE, vol. 47, pp. 1213-1218, Jul. 1959.

[22] D. Ham and A. Hajimiri, "Concepts and methods in optimization of integrated LC VCOs," IEEE J. Solid-State Circuits, vol. 36, no. 6, pp. 896-909, Jun. 2001.

[23] H. Brauns and W. Konrath, "Ultra low phase noise parametric frequency divider for highest performance microwave-and milimeterwave frequency sources," in Proc. Eur. Microw. Conf., Oct. 1995, vol. 2, pp. 1155-1158.
[24] U. L. Rohde, Microwave and Wireless Synthesizers. Theory and Design. New York: Wiley, 1997.

[25] C.-C. Ho et al., "0.13- $\mu \mathrm{m}$ RF CMOS and varactors performance optimization by multiple gate layouts," IEEE Trans. Electron Devices, vol. 51, no. 12, pp. 2181-2185, 2004.

[26] W. F. Andress and D. Ham, "Standing wave oscillators utilizing waveadaptive tapered transmission lines," IEEE J. Solid-State Circuits, vol. 40, no. 3, pp. 638-651, Mar. 2005

[27] S. A. Wartenberg and J. R. Hauser, "Substrate voltage and accumulation-mode MOS varactor capacitance," IEEE Trans. Electron Devices, vol. 52, no. 7, pp. 1563-1567, Jul. 2005.

[28] T. Luo and Y. E. Chen, "A 0.8-mW 55-GHz dual-injection-locked CMOS frequency divider," IEEE Trans. Microw. Theory Tech., vol. 56, no. 3, pp. 620-625, Mar. 2008.

[29] B. Razavi, Design of Integrated Circuits for Optical Communications. New York, NY: McGraw-Hill, 2003.

[30] B. Kim, L. Kim, S. Byun, and H. Yu, "A 20 Gb/s 1:4 DEMUX without inductors and low-power divide-by- 2 circuit in $0.13 \mu \mathrm{m}$ CMOS technology," IEEE J. Solid-State Circuits, vol. 43, no. 6, pp. 541-549, Jun. 2001.

[31] A. Hajimiri, "Noise in phase-locked loops," in Proc. IEEE Southwest Symp. Mixed-Signal Circuits, Feb. 2001, pp. 1-6.

[32] J. Lee, M. Liu, and H. Wang, "A 75-GHz phase-locked loop in 90-nm CMOS technology," IEEE J. Solid-State Circuits, vol. 43, no. 6, pp. 1414-1426, Jun. 2008.

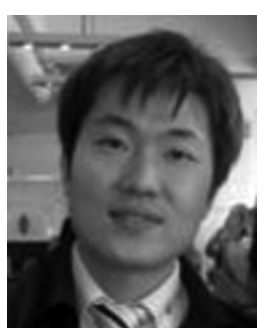

Wooram Lee (S'07) received the B.Sc. and M.S degrees in electrical engineering from the Korea Advanced Institute of Science and Technology (KAIST), Daejeon, Korea, in 2001 and 2003, respectively. He is currently working toward the Ph.D. degree at Cornell University, Ithaca, NY.

He was a Research Engineer with the Electronics and Telecommunications Research Institute (ETRI), Daejeon, Korea, where he worked on optical transceivers and links for WDM-PON from 2003 to 2007. His research interests include high-performance RF IC design, based on nonlinear electronics for low-noise parametric amplification, oscillation, and terahertz pulse generation.

Mr. Lee was the recipient of the the IEEE Solid-State Circuits Predoctoral Fellowship for 2010-2011 and the Samsung Graduate Fellowship for 2007-2011. He was also a recipient of the Best Paper Award of the IEEE Radar Conference in 2009 and the Silver Medal at the National Physics Competition in 1996.

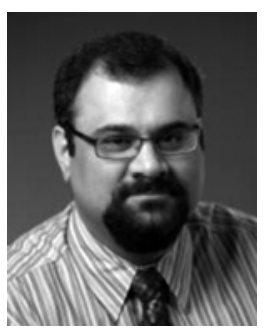

Ehsan Afshari (M'07) was born in 1979. He received the B.Sc. degree in electronics engineering from the Sharif University of Technology, Tehran, Iran, and the M.S. and Ph.D. degrees in electrical engineering from the California Institute of Technology, Pasadena, in 2003 and 2006, respectively.

In August 2006, he joined the Faculty of Electrical and Computer Engineering, Cornell University, Ithaca, NY. His research interests are high-speed and low-noise integrated circuits for applications in communication systems, sensing, and biomedical

devices.

Prof. Afshari serves as the chair of the IEEE Ithaca section, as the chair of Cornell Highly Integrated Physical Systems (CHIPS), and as member of the Analog Signal Processing Technical Committee of the IEEE Circuits and Systems Society. He was the recipient of the National Science Foundation CAREER Award in 2010, the Cornell College of Engineering Michael Tien Excellence in Teaching Award in 2010, DARPA's Young Faculty Award in 2008, and Iran's Best Engineering Student Award by the President of Iran in 2001. He was also the recipient of the Best Paper Award at the IEEE Custom Integrated Circuits Conference (CICC), September 2003, the First Place Award at the Stanford-Berkeley-Caltech Inventors Challenge, March 2005, the Best Undergraduate Paper Award in the Iranian Conference on Electrical Engineering, 1999, the Silver Medal in the Physics Olympiad in 1997, and the Award of Excellence in Engineering Education from the Association of Professors and Scholars of Iranian Heritage (APSIH), May 2004. 